\title{
The Place of Concept in Human Cognitive Process of Perception: Why the Conceptualists Cannot Be Right?
}

\author{
Emmanuel Ola Akintona \\ Department of Communication and General Studies, College of Agricultural Management and Rural \\ Development, Federal University of Agriculture, Abeokuta, Nigeria \\ Email: emmanuelakintona@gmail.com
}

Received 21 January 2015; accepted 9 February 2015; published 10 February 2015

Copyright (C) 2015 by author and Scientific Research Publishing Inc.

This work is licensed under the Creative Commons Attribution International License (CC BY). http://creativecommons.org/licenses/by/4.0/

(c) (7) Open Access

\begin{abstract}
There have been so many controversies in the meaning of concept and particularly its place in the cognitive process of perception. The conceptualists, particularly, John McDowell, D. W. Hamlyn, Bill Brewer and Sonia Sedivy, argue that the content of perceptual experience is always in a kind of relation with propositional attitude such that beliefs, judgments, hopes and aspirations are instantaneously captured in perception. If this is granted, then, it becomes difficult to admit the possibility of non-conceptuality in perception. But, on a critical look at the conceptualists' arguments and deductions, we discover that the conceptualists conflate sensation with perception and concept formation. In view of this, this paper examines and does a critical analysis of the meaning of concept with the belief that if its place in the cognitive process of perception is determined and ascertained, the long standing problem about the nature and characterization of the content of human perceptual experience will automatically dissolve. Whilst distinguishing and separating sensation from perception, the paper establishes that concept-formation is not generic to perception and that there is a place for non-conceptuality in perception. This paper employs conceptual analytical tools to explain the place of concept, sensation and perceptual experience in the cognitive process of perception and thus establishes the truism of non-conceptuality in perception.
\end{abstract}

\section{Keywords}

Language, Concept, Sensation, Perceptual Experience, Cognition and Knowledge

\section{Introduction}

John McDowell, D. W. Hamlyn, Bill Brewer and Sonia Sedivy are among the strong conceptualists who claim 
that concept is spontaneously structured on perceiving the world. For them, the content of perceptual experience is always in a kind of relation with propositional attitudes such that beliefs, judgments, hopes and aspirations are implicitly part of it. They contend that concepts are immediately derived in perception and dependent on experience, hence, perceptual experience is concept-bound.

If having concepts means cognizing experience, then, perception would imply a completed process of perception different from mere sensation. The conceptualists argue that not until one is able to form concept about one's experience, one cannot be said to have truly experienced the world. For them, experience is strictly tied to concept and any experience that is not conceptual is not genuine.

But, looking at this argument critically, one is forced to make distinction among perception, sensation and experience. And the question is asked, is experience the same thing as perception? To experience the world is to sense the world and to perceive it is to conceptualize it. To make clear this distinction some scholars employ "concept" in such a way as to warrant making a distinction between sensation and perception. The two, they claim, are not the same and as such, should not be conflated. Thomas Reid, for instance, says, "sensation has no object other than itself, while perception has physical things as its object and also involves concept and beliefs about them" (Hamlyn, 1994: 147). By this, he means that sensation produces no concept at all but perception necessarily does. One would have expected the conceptualists to adopt this distinction and accept non-conceptuality as something derivable from sensation at least. But, they insist that perception must be related to the external world in some ways, hence, must be conceptual. Since the perceiver must have some kinds of beliefs about his experience no matter what, his experience cannot but be conceptual.

This paper does a critical analysis of the word, "concept" both from the traditional and contemporary perspectives, determines its placein perception and shows how it relates to sensation. Some conceptualists' arguments on the nature and characterization of the content of perceptual are also examined. The point being made here is that if the place of concept and concept-formation is determined in the process of perception then, ascertaining the possibility of non-conceptual content in perception becomes unproblematic.

\section{The Classical Notion of Concept}

Philosophers have divergent views on what concept is and possibly its place in perception. The notion, "concept", and its meaning have been loosely employed in epistemology until the modern period when it has to be explained in relation to perception. The controversy surrounding its meaning explains, in part, the rivalry in epistemology between the rationalists and the empiricists. The concept-rationalists hold that concepts are innate, while the concept-empiricists argue that concepts are derived from experience. Going by the former, concepts are independent of, and are derived prior to, experience. Plato claims that there are certain things we know which we do not necessarily perceive with the senses, such as honour, similarity, goodness, badness and so on. He argues that "Forms or Ideas" are known through dialectical reasoning and not by sense-perception because they are derived prior experience. For him, the soul was formerly acquainted with ideas/forms of things before it became imprisoned in the body: the soul already possessed the knowledge of concepts of things before its encounter with the phenomena world. It means, therefore, that concepts are innate. This conclusion, however, raises some epistemological questions that needed serious attention.

If concepts are inborn, it would imply, for instance, that a man born blind would have concepts as much as a non-blind man. But can a man born blind possess the concept of redness if he had not initially experienced it? The concept-rationalists claim that some concepts like cause and effect and the idea of God are conceivable outside experience (Hospers, 1956: 102). Even though this argument seems naive in the modern period, our concern is to show that concepts and concept-formation are separable from experience and independent of it so that the notion of non-conceptual content in the cognitive process of perception is established.

In the seventeenth and eighteenth centuries, the words "idea" and "concept" were used interchangeably, until "idea" became extended in the twentieth century to include feelings and imaginations. For example, the expression, "I have the idea that someone is watching me" simply translates to mean "I have the impression that someone is watching me". David Hume (1711-1776) was the first to make a clear distinction between "impression" and "idea". He explains idea as "a weak copy or a shadow of an impression". By this, he means, impression is what we actually perceive not ideas, He espoused,

if I have a look at a green tree, what I have is a sense-impression of green but when I close my eyes to imagine something green, all I have is the idea of green (Hospers, 1956: 102). 
Hume relates impression to perception and ideas to imagination. He defines concepts in terms of image formation in the mind, and since no image can be formed in the mind without perceiving, he concludes that one must first have an impression (sensation) before having the idea (concept). So, for Hume, impressions precede ideas, hence, sensation comes before concepts and is separable from it. Therefore in perception, what we first experience is impressions and not ideas. And without impression it means that the process of thought cannot take place because what we see is what we conceptualize. From this line of argument, we observed that Hume indeed conflates impression and experience with sensation.

Hume's argument is also in consonance with Immanuel Kant's popular dictum "Thoughts without content are empty, intuitions without concepts are blind" (Kant, 1997: A19/B33). Perhaps, the idea that "impression precedes idea” informs Hume’s popular thesis, "No impression, No idea”. He asserts,

if anyone claims that he has some ideas, we only need to ask him, from what impression is that supposed idea derived? And if it is impossible to assign any, this will serve to confirm our suspicion... (Hospers, 1956: 113).

The implication of this is that a man who has never experienced pain can never have idea of pain and a man who has never experience fear cannot have the idea of fear, while the lady who has never experience sexual sensations cannot have the idea of sexuality even though she might have observed people having sex.

Locke further distinguishes between simple and complex ideas. For him, simple idea is the simplest unanalyzable fraction of perception such as red, sweet, pain, etc. while formulating the idea of a "golden mountain and black roses" belongs to the category of a complex idea. He claims that the human mind possesses the ability to put together, through the faculty of imagination, the combination of simple ideas that have already been acquired through particular experiences to form new but complex ideas. Therefore, no human mind can create simple ideas without having a particular experience. It therefore means that any one who has never seen the colour red or ever feels pain before can never have the concept. He asserts:

Nothing... seems more abounded than the thought of man, which not only escapes all human power and authority but is not even restrained within the limits of nature and reality. To form monster, and join incongruous shapes and appearances, costs the imagination no more trouble than to conceive the most natural and familiar objects (Hospers, 1956: 104).

The creative power of the human mind amount to no more than the faculty of compounding, transposing, augmenting or diminishing the materials furnished it by the senses and experience. So, when we imagine "a golden mountain", all that we have done is only to join two consistent ideas, "gold" and "mountain", with which we were formerly acquainted.

Hospers (1956: 108) suggests four ways of characterizing “concepts”. First, if the subject can "apply the word $\mathrm{x}$ correctly". Second, if the subject "is able to define x correctly". Third, if the subject can "distinguish $\mathrm{x}$ from $\mathrm{y}$ and $\mathrm{z}$, and indeed, everything that is not an $\mathrm{x}$ ". Fourth, if the subject has "some criterion-in-mind... having some kinds of 'mental content' independent of words and distinguishable from objects". The second criterion seems too narrow to be accepted because there are many words we seem to know very well that cannot be defined appropriately. Even, the compilers of dictionaries sometimes have difficulties with words like "red" which are really simple ideas but not definable.

To define concepts in terms of the ability to distinguish between things will amount to claiming that a child who is able to distinguish cats from dogs and pigs and all other things, has the concept of what a cat is even though he cannot analyze what it takes for an animal to be a cat. Would it then mean that a dog which can distinguish a cat from a bird possesses the concept even though it has no word for it? The ability to distinguish between $\mathrm{x}$ and $\mathrm{y}$ is not enough to ascertain whether one has the concept or not. Defining concepts as having some criterion-in-mind will also amount to suggesting that the mind can have some kinds of "mental content" independent of words distinguishable from objects. For example, one may have a concept of something that is a reptile, larger than an elephant that flies through the air. At least, the fact that such a creature is not in existence does not foreclose one from having such a concept. And to define concepts in terms of correct application of words will amount to reducing it to mere linguistic achievement. Therefore, to have the concept of redness or orange ness will mean the ability to apply the word "red" or "orange" correctly anytime it is used in a language.

However, if having concepts is the function of correct-usage of words in language, then which one comes first, Language or thought? Thought cannot be functional without "sensing”. It only strives midst of language. Can 
language ever get started without thought? We know human develop his/her senses early enough before language was developed. Human indeed uses his/her senses (introception and extroception) to experience the phenomena world before concepts are ever developed. Locke (1993: 31) argues "the phonemes of our mother's tongue seem to have a start in utero but sentences emerge only after a year or two of childhood".

Scientific investigation revealed that by three years, most children "glibly generate sentences, and have the basic grammar right" (Davidson, 1997: 19). Pinker (1995: 18) also posits that "language is not a cultural artifact that we learn the way we learn to tell time... Instead, it is a distinct piece of the biological make-up of our brains”. This is an indication that when we are to comprehend sentences as the stream of words is transparent and we see through to the meaning automatically. On this note, Pinker (1980: 307) calls language a "mental organ" only to show that there is a strong connection between language and thought and to establish the existence of an innate or genetically programmed internal language, called, mentalese ${ }^{1}$ (the language of thought). Pinker argues that there is an inner language that emerges as part of our genetic heritage prior any spoken language. $\mathrm{He}$ asserts,

mentalese does not depend on the development of language, instead language depends on it. Given the universal grammar that is wired in every human, the connectedness of words... reflects the relatedness of ideas in mentalese. This resolves the problem of taking an interconnected web of thought in the mind and encoding them as a string of words... For the child, the unknown language is English, Japanese or Arabic, the known one is mentalese (Pinker, 1995: 101).

Pinker compares the child to Quine's radical translator. The only difference in his comparison lies in the fact that the child simply knows automatically what idea is represented to him in mentalese by the words he hears but the radical translator does not. To Pinker, the mentalese has priority over language because it is acquired first. He argues that since we do not think in English or Yoruba, then it becomes necessary for thinking to have some salient medium in the brain such as the language of thought or mentalese.

Language is learned and is characteristically dynamic, yet it develops closely with thought: it is quite closely related to, even though different from, thought. In other words, if concept is entailed in language and better understood and expressed in language, then it cannot be innate. Among human developmental stages, thought is primary while language is secondary. So, thought necessarily precedes language.

\section{The Contemporary Notion of Concept}

Concept in the contemporary time is conceived as an abstract idea representing something in a language. Stalnaker (2004: 3) defines concept in terms of:

an abstract idea or a mental symbol which is typically associated with a corresponding representation in language or symbology that denotes all of the objects in a given category or class of entities, events, phenomena, or relationships between them.

In this sense, conceptisnot only an abstract idea representing something in a language but something universal in nature which can be applied to all things in their extension. As words are basic semantic elements toa sentence so are concepts basic elements toa proposition (ibid). Concepts are bearers, not agents of meaning. Therefore, a single concept can be expressed in a number of languages. For example, "dog” can be expressed as dog in English, Aja in Yoruba, Kare in Hausa, chien in French, or perro in Spanish. This fact makes translation possible without alteration in meaning. Translations are based on the kind of concept formed about one's experience and not particularly on the structure of a language perse. Stalnaker further posits,

the notion of perceptual content begins with the idea that what is said in a speech act (the proposition expressed), can be abstracted from two different aspects of the way it is said: first, from the means used to express it, and second, from the force with which it is expressed (ibid).

Apart from this, a proposition can be the content of an assertion in one context and of a supposition (a component of a disjunctive assertion) or a request in another. It is possible that the content of what is expressed in a speech act is the same as the content of the mental state of a different kind. Just as what is said can be separated

${ }^{1}$ Mentalese is a genetically programmed language of thought. According to this theory, this inner language is not learned but emerges as part of our genetic heritage, and it is prior to any spoken language. The essential feature of mentalese is that it does not depend on the development of language but vice versa. See Donald Davidson, “Seeing through Language” Supplement to Philosophy, vol. 42 (1997): 19. 
from how it is said, so what is thought is also separable from its mental representation or the kind of mental state (belief, wish, tacit presupposition, hope or fear) the proposition specifies. Just as you and I might say the same thing even though you say yours in French and I say mine in English. It seems prima facie reasonable then to say that when something merely looks to me in a certain way, even though I do not really believe it is truly that way, it does not rule out the fact that I still have some representational content in my perceptual experience. The fact still remains that I am in one perceptual state regardless of whether my belief is correct or not.

Rand (1990: 15-28) explains concept in line with the principle of objectivism where concept is taking as something "derivable from and do refer to the facts of reality". So, any concept that lacks correspondence with objective facts is not genuine at all. For him, "concepts are formed in the context of knowledge available”. So, a young child who is able to differentiate dogs from cats and chickens, need not explicitly differentiate them from deep-sea tube worms, or from other types of animals not yet known to him, to form the concept of "dog". He explains consciousness in terms of identification and by this, he means that "reason" is "the faculty that identifies and integrates the material provided by the senses”" ${ }^{2}$. For the empiricist, the mind at birth is tabula rasa, therefore the senses serves as a pre-condition for conceptualization. It now depends on how sophisticated our conceptual capacity is that determines what it can comprehend and interpret. Anything that falls outside the experience (culture, biological make-up or an already formed belief) engendered by the subject remains non-conceptual for him.

What concept one form can then be narrowed down to one's ability to organize and interpret the repertoire of information deposited into the mind by the senses. Concepts are mere extrapolations of the vast repertoire of the perceptual fields of the perceiver. The conglomeration of these fields is integrated into a unified mental unit (concept) which is later symbolized in words or statements in a language.

\section{The Conceptualist Account of Concept in Perception}

What exactly do we mean by "conceptual content"? It is a content of a kind that can be ascribed to judgment and belief. By this, conceptual contentcan then be understood as that which has identities conforming to Frege's criterion of identity for sense and reference. Concepts, by this criterion, are constituent of that intentional content that can be the complete, truth-evaluable, component of judgment and belief. The contents of "p" and "q" are distinct if and only if it is possible for someone to rationally judge that "p" without judging that "q".

The conceptualists argue that no representational content is devoid of recognition or demonstration hence all perceptual contents are necessarily conceptual. John McDowell (1994) sees experience as strictly a conceptual achievement. For him, every experience is subsumable under concept, whether as belief or judgment and any experience that cannot be put under concept should be disregarded as relevant to perception. For him, all forms of experiences are intentionally directed and belief-bound, so, he cannot imagine any experience devoid of belief. He asserts, "nothing can simply be a reason for a belief except another... belief" (McDowell, 1994: 44). Therefore, only beliefs can be reason for other beliefs (ibid: 63). But, if the only rational ground for a belief is other beliefs, then would we not be running the risk of a vicious circle? And perhaps, what would be the role of sensation in perceptual experience? We can implicitly deduced from McDowell's line of thought the he endorses the fundamental rationalists' insight which suggests that "to be aware of something in the sense that such awareness can serve as evidence for beliefs amounting to knowledge is to bring it under a concept”. Corroborating this view, Hamlyn (1994: 144) can also be summarized as saying that "concepts are necessarily and instantaneously captured in perception".

McDowell refused to see any reason why perceptual experience should be divided into non-conceptual and conceptual content since they are of one and the same experience. He argues that the process of judgment does not introduce a new kind of content into one's perceptual experience; instead, it "simply endorses that conceptual content or some of it that is already possessed by the experience on which it is grounded" (McDowell, 1994: 49). And if this is the case, then McDowell's argument is self-contradictory and faulty. However, McDowell further vindicates the non-conceptualists' account accepting that experience is more fine-grained than the concepts, beliefs and judgments we possess because the entire content of our perceptual experience is not always endorsed by our beliefs and judgments (ibid). If the non-conceptualist's claim is true, then McDowell's position that the content of perceptual experience is necessarily conceptual would be wrong.

${ }^{2}$ For more of Rand's Theory of concepts see Rand, 1990: 15-28, Kelly, David “A Theory of Abstraction” and “The Psychology of Abstraction”, Cognition and Brain Theory vol. vii, no 3 and 4 (Summer/Fall 1984) and Rasmussen, Douglas, B., "Quine and Aristotlelian Essentialism”, The New Scholasticism 58 (Summer, 1984). 
Giving McDowell the second reading will expose us to more inconsistencies in his argument on the status of the content of perceptual experience. Sometimes, he accepts that non-conceptuality is possible but that it is not relevant to perception, and at another time, he gives the impression that non-conceptuality is not possible at all. He admits two kinds of beliefs which are acquired non-inferentially: one, by the exercise of reliable dispositions to respond differently to stimuli or reporting elements of the causal chain which culminates in the report; and two, by mere non-inferential observation. In genuine perception, he argues that the belief is the result of endorsing the content of a perceptual experience but in mere observation, the belief is acquired blindly. He claims that under the right circumstance, one just finds himself with the belief in question. However, this sort of belief formation is not a case of facts becoming visible and generally perceptible to us. Although these beliefs are noninferentially elicited from the believer by environmental stimuli, the warrant for those beliefs is in an important sense inferential. The believer's justification for beliefs of this sort depends on drawing conclusions from an antecedent claim of reliability. In this respect, he espoused that the believer himself is in the same situation with the observer.

McDowell does not construe perceptual experience as something involving the sort of endorsement characteristic of judging or believing but of content that is judgeable and believable which means that the content of perceptual experience is itself not judgments or beliefs. So, when a perceiver advances from perceptual experience to judgment or belief, the experience only serves in the capacity of justifying the resulting commitment, no more no less. This signifies McDowell's endorsement of the Fregean approach, which construes facts as true thought: "thoughts" not in the psychological sense of thinking, but in the semantic sense of the contents that are thought or that which is thinkable. This argument seems to rule out the possibility of perceptual mistakes, because we sometimes cannot tell the difference between the cases in which we are having a perceptual experience whose content tallies with reality and cases where there is no such reality to be perceived at all.

Traditionally, this argument has been attacked from the perspective of the famous "Argument from Illusion" where the perceiver has perceptual experience and the same content common to both veridical and non-veridical cases $^{3}$ (see Argument from illusion). McDowell's objection to making distinction between conceptual and nonconceptual content is however not epistemological but about semantics. His doctrine of semantic empiricism is only to show that if we can make this a feature of our thought and talk intelligible for perceptual experience, then we can make it intelligible for any claim or belief. For him, the only thing a veridical perceptual experience and a corresponding hallucination have in common is that their subject cannot separate them apart but that their experience differs.

McDowell (1994) declares that perceptual experience is "immediate" in the same sense that the conceptual abilities are required, so, there is no need for making any distinction between them. He claims that concepts are instantaneously acquired in perception and that the conceptual content for making judgment by inference is the same as the perceptual content for observation. The only visible difference lies in the wrong application of concepts when we expressed them in language. In truth, the ways in which concepts are brought into perception sometimes fall short of judgment or belief but the same content is presented to the potential knower for endorsement. An act of judgment is only required to endorse it. So the problems arising from us by the fact are not judgment but only petitions from our judgment. This explanation is necessary because McDowell does not just want to make any distinction in our perceptual experience so as to make intelligible the sense in which we are rationally responsible for our perceptual judgments. Notwithstanding, his argument here seems inadequate because it is difficult to see how contents are judgeable and yet not endorse by judgment. A picture merely entertaining judgeable content, awaiting the exercise of an act-of-will without judgment is here questionable.

Judging knowledge from a dispositional point of view, we can distinguish between animals and humans. A parrot could be taught to respond to red things by uttering the noise "that is red" but it would not be saying or claiming that anything was red. To lay claim on something is to make an assertion about that thing. To make a claim requires commitment to provide evidence for such a claim which no other animal can provide. There is a wide difference between what a reporter can be trained under some circumstances to say or do and what concepts he/she possesses by applying those concepts to responsive dispositions.

Strictly going by McDowell it will mean that to be aware of something is just to apply concepts to it, which is, making a judgment or undertaking a doxastic commitment regarding the awareness. Awareness deserves to be called "immediate" just in case it is not the product of a process of inference. Therefore, beliefs acquired

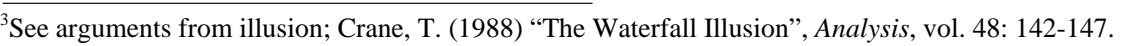


non-inferentially, especially through the exercise of reliable dispositions to respond differently to stimuli of a certain sort, is yet conceptual even though it embodies immediate awareness of the items reported. In McDowell's view, this is the only sense of "immediate awareness" where we can understand our perceptual knowledge of the world around us.

A reliable non-inferential response to events does not necessarily mean that one has concepts of such events. Observational/non-inferential knowledge should be distinguished from cases of genuine perceptual knowledge. What McDowell often refers to as knowledge derived from perception can be classified under observational knowledge. But at times, when we see colours or shapes, we have perceptual experiences corresponding to the judgment from which we can go on to make or form beliefs without having genuine knowledge about them. We sometimes respond blindly and still trust such blind responses only to discover later the truth about such experiences.

Brewer (2004: xx) claims that "perceptual experiences justify beliefs" and as such "sense experiential states provide reasons for empirical beliefs". He, therefore, conceives perceptual experience as that with rational relations to judgment and beliefs to the extent that its spontaneity is already implied in its reception. This will only apply if we take experience as having conceptual content in the sense McDowell (1994: 162) conceives it. Sellars (1956: 111) also sees concepts as the sole responsibility of the correct use of words. For her, having concept involves mastering the use of words. Therefore, if a word is properly used, it is believed that one has the concept. So, for Sellars, no one can understand the concept "red" unless he knows what it is for things to look "red" and make an appropriate use of the word.

Davidson (1997: 15-27), in "Seeing through Language", describes language as a means by which we talk about the world but not the act of conceptualizing it. If this is the case, then language is a vehicle for conveying concepts and not their maker. Language may not reproduce for the mind the accurate records of the world; if it does, how come cases of distortions or illusions? He defines language as,

a convenient human skill which we use in coping with one another in our common terrestrial setting. Without it we would not think of things, as we do. But it does not follow, of course, that we never perceive how the world really is, as Kant thought, or that every view is necessarily distorted, as Bergson and many other have held (ibid: 15).

We can then make a deduction from the above quotation that perception is quite separable and independent of language. The absence of language does not really rule out perception. If we can, at least in principle, isolate some given aspects of perceptual experience, which cannot be shaped by the mind, how do we then classify such aspect? Perhaps, it should be best described as non-conceptual.

\section{Sensation and Perception}

Sensation simply refers to the process of sensing the environment through touch, taste, sight, sound, and smell. Information gathered from sensation is sent to the brain in raw form where perception comes into play. Perception is the way we interpret the signals from sensations and make sense out of them. However, percepts are not spontaneously interpreted or understood while perceiving the environment, depending on the level of development of the mind and already accrued experience, there are always some left-over (non-conceptual content). Sensation is a passive process whereby information from the outside (the environment) are brought into the body and brain: it is a passive process because the subject need not be conscious of the "sensing" process, but for perception, it is a deliberate and conscious phenomenon. The subject is conscious and actively involved in the process of perception. Perception is therefore a deliberate attempt by the subject in selecting, organizing, and interpreting data brought to bear on brain by the senses ${ }^{4}$.

In human perception, a large number of information is sensed every minutes ranging from room temperature to the brightness of lights in the room but are all recognized instantly? We sometimes hardly notice radio waves, $\mathrm{x}$-rays or other microscopic parasites crawling on our body; not all odours or taste do our senses notice because of the limited pattern of our thresholds. For an absolute threshold, everything becomes noticeable to the senses. For example, at the softest of sound, one can hear and at the slightest touch, one can feel (some animals are uniquely gifted in sensing some percepts) but anything less than this goes unnoticeable to the senses.

\footnotetext{
${ }^{4}$ See Psychology of Sensation and Perception in www.alleydog.com/101notes/s\&p.html. Reading Gestalt psychology will also expose us much better to how and why we perceive things.
} 
For sensation to occur, sensory organs absorb energy from the physical stimulus in the environment, the receptors then convert this energy into neutral impulses and send them to the brain. But for perception to occur, the brain organizes the raw data collected from sensations and translates them into something meaningful. Meaningful information or concepts derivable in the brain are influenced by psychophysics, that is, the study of how physical stimuli are translated into psychological experience. This majorly comprises selective attention and perceptual expectancy. The former is influenced by what motivated perception while the latter is influenced by our past experience, culture and biological make-up. For example, the subject does not expect a cow to fly or a tree to speak when he perceives them. Better still, the subject may observe a painting and not really understood the intended message of the artist until someone explains to him. There and then, things that were formerly oblivion may become obvious and the intended message unravels.

\section{Conclusion}

Even though sensation and perception are complementary, they play different roles in cognition. While sensation is the process by which the senses (touch, taste, sight, sound and smell) gather data from the environment and are sent to the brain for interpretation, perception is an already processed data in the brain that represents information about the perceived environment. Perception is simply the way that we interpret sensations and conceptualize it. Kant (1997: A51/B76) corroborating this dichotomy argues that sensibility is independent of understanding. He describes understanding as a logical, discursive and proposition-forming capacity of the mind which produces concepts as output and sensibility as the affective, perceptual and imaginational capacity of the mind that produces intuition as output. If this is granted, then, sensibility and understanding are separable. Therefore, if it is possible to conceive the two separately, then the contention whether non-conceptuality is possible in perception or not, automatically dissolves. At least, what the perceiver forms as concept depends on his past experience, culture and biological make-up, and since not all that are sensed are conceptualizable or immediately conceptualized, there is room for non-conceptuality in perception, hence, the conceptualists cannot be right.

\section{References}

Brewer, B. (2004). Perception and Conceptual Content. In E. Sosa, \& M. Steup (Eds.), Contemporary Debates in Epistemology (pp. 89-112). Oxford: Basil Blackwell.

Davidson, D. (1997). Seeing through Language. Supplement to Philosophy, 42, 15-27. http://dx.doi.org/10.1017/S135824610001016X

Hamlyn, D. W. (1994). Perception, Sensation and Non-Conceptual Content. The Philosophical Quarterly, 44, 139-153. http://dx.doi.org/10.2307/2219737

Hospers, J. (1956). An Introduction to Philosophical Analysis. London: Routledge and Kegan Paul Ltd.

Kant, I. (1997). Critique of Pure Reason (A: 1881 \& B: 1887). Cambridge: Cambridge University Press.

Locke, J. (1993). An Essay Concerning Human Understanding. London: Everyman's Library. Dent/Dutton; First Published in 1690 .

McDowell, J. (1994). Mind and World. Cambridge, Mass.: Harvard University Press.

Rand, A. (1990). Objectivism. http://en.wikipedia.org/wiki/Concept

Pinker, S. (1995). The Language Instinct: How the Mind Creates Language. http://en.wikipedia.org/wiki/The_language_instinct

Sellars, W. F. (1956). Empiricism and the Philosophy of Mind. In H. Feigl, \& M. Scrivens (Eds.), Minnesota Studies in the Philosophy of Science (Vol. 1, pp. 253-329). Minneapolis: University of Minnesota Press.

Stalnaker, R. (2004). What Might Non-Conceptual Content Be? Philosophical Issues, Villanueva. Atascadero (2003) CA: Ridgeview. Reference to the Reprinting in Gunther. http://en.wikipedia.org/wiki/Concept 
Scientific Research Publishing (SCIRP) is one of the largest Open Access journal publishers. It is currently publishing more than 200 open access, online, peer-reviewed journals covering a wide range of academic disciplines. SCIRP serves the worldwide academic communities and contributes to the progress and application of science with its publication.

Other selected journals from SCIRP are listed as below. Submit your manuscript to us via either submit@scirp.org or Online Submission Portal.
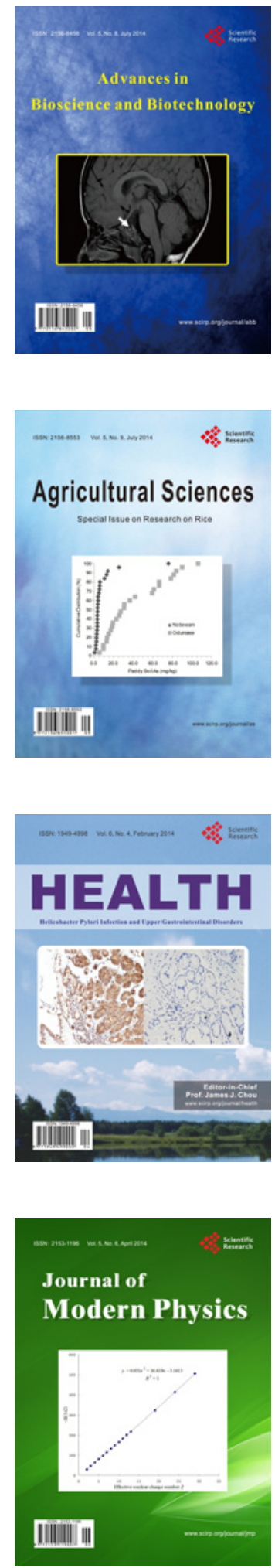
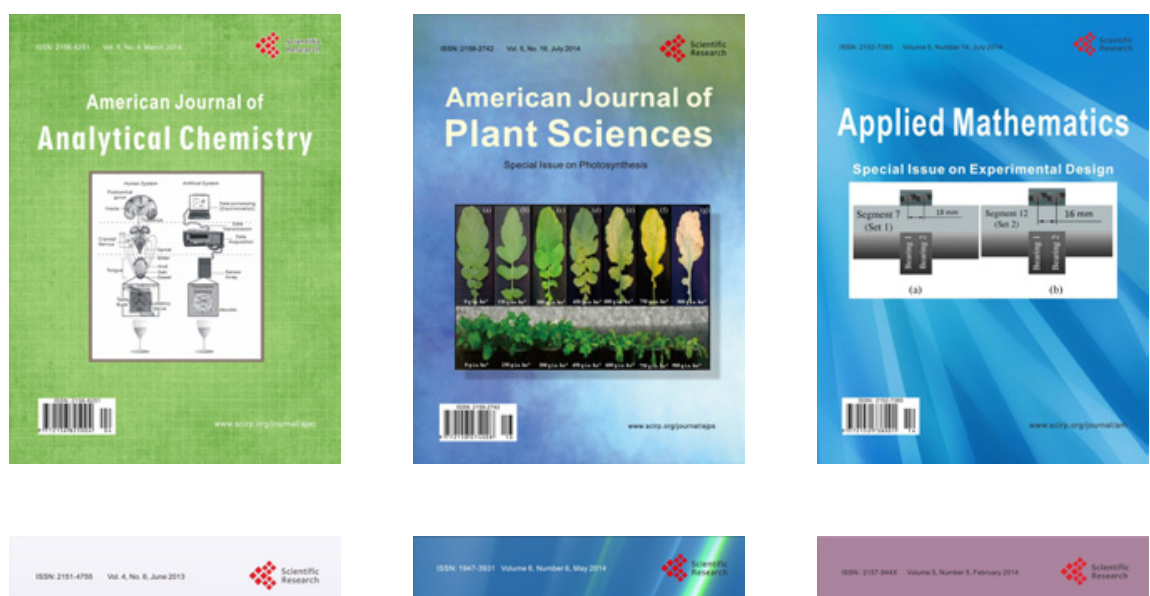

Creative Education
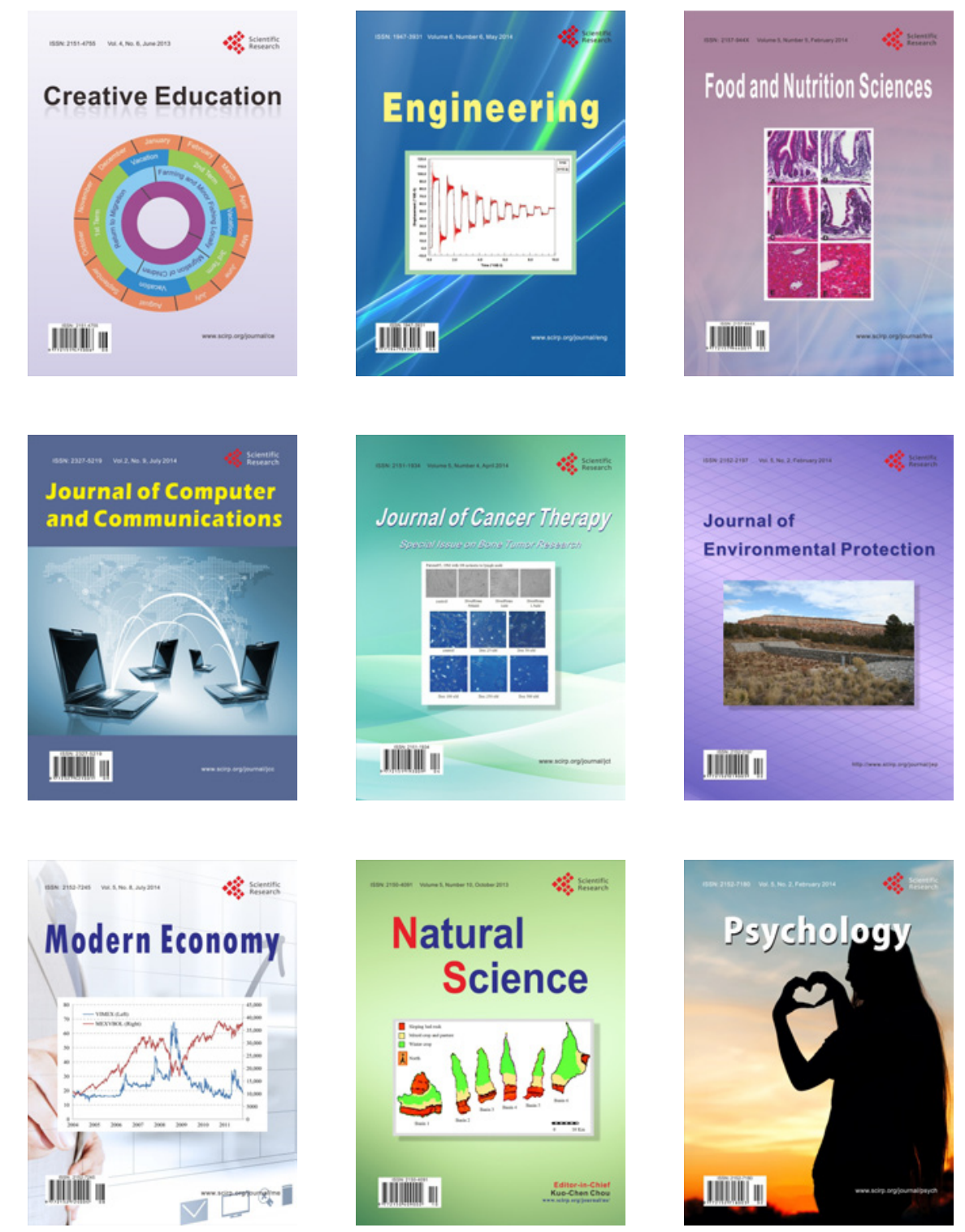